\title{
Mobile Data Mining for Intelligent Healthcare Support
}

\author{
Pari Delir Haghighi, Arkady Zaslavsky, Shonali Krishnaswamy, Mohamed Medhat Gaber \\ Center for Distributed Systems and Software Engineering \\ Monash University, Australia \\ \{pari.delirhaghighi, arkady.zaslavsky, shonali.krishnaswamy,, mohamed.gaber\}@infotech.monash.edu.au
}

\begin{abstract}
The growth in numbers and capacity of mobile devices such as mobile phones coupled with widespread availability of inexpensive range of biosensors presents an unprecedented opportunity for mobile healthcare applications. In this paper we propose a novel approach for Situation-Aware Adaptive Processing (SAAP) of data streams for smart and real-time analysis of data. The implementation and evaluation of the framework for a health monitoring application is described.
\end{abstract}

\section{Introduction}

Recently, innovations in mobile communications and low-cost of wireless biosensors have paved the way for development of mobile healthcare applications that provide a convenient, safe and constant way of monitoring of vital signs of patients. A key in the provision of mobile healthcare services is the issue of using technological innovation to support continuous monitoring of patient conditions, providing a degree of self-diagnosis and enabling effective real-time decision making to reduce fatalities. Ubiquitous Data Stream Mining (UDM) techniques [1] such as lightweight, one-pass data stream mining algorithms [2-3] can perform real-time analysis on-board small/mobile devices while considering available resources such as battery charge and available memory. However, to perform smart and intelligent analysis of data on mobile devices, it is imperative for adaptation strategies to factor in contextual information.

Contextual information can be related to a network, application, environment, process, user or device. As a meta-level concept over context we define the notion of a situation that is inferred from contextual information [4]. Situation-awareness provides applications with a more general and abstract view of their environment rather than focusing on individual pieces of context. Situation-aware adaptive data stream mining leverages the full potential of UDM by going beyond mere available resources and can enable, if not guarantee, the continuity and consistency of the running applications.

In real-world, situations evolve and change into other situations (e.g. 'healthy' changes to 'hypertension'). Changes that occur between situations are also good indicators of situations that may emerge - albeit with some vagueness and uncertainty. To enable situation-awareness in mobile healthcare applications, it is important for the situation modeling and reasoning approach to represent uncertainty and vagueness associated with health-related situations.

Reviewing recent works in mobile healthcare reveals that most of these projects [5-8] have mainly focused on using, enhancing or combining existing technologies and context-aware projects [9-13] mostly deal with a limited scope (i.e. not applicable to other context-aware scenarios). In mobile healthcare computing, a general approach for modeling and reasoning about uncertain, health situations and performing smart and cost-efficient analysis of data in real-time has not been introduced and is an open issue.

In this paper we propose situation-aware adaptive processing (SAAP) of data streams for mobile healthcare applications. The novelty and contribution of this project are as follows: i) situation-awareness is achieved by Fuzzy Situation Inference (FSI) that combines fuzzy logic principles with the Context Spaces (CS) model, a formal and general context modeling and reasoning for pervasive computing environments. The strengths of fuzzy logic for modeling of vague situations are combined with the CS model's underlying theoretical basis for supporting context-aware pervasive computing scenarios; ii) SAAP incorporates situation-awareness into data stream mining and provides gradual tuning of data streaming parameters according to occurring situations and available resources. This approach improves data stream mining operations in an intelligent and costefficient manner. The SAAP approach enables continuity and consistency of running operations that are of high important for health monitoring applications that deal with sensitive and critical data. 


\subsection{A Scenario}

John has had a heart attack and is released from hospital but there are concerns that he might be susceptible to another heart attack and is also experiencing blood pressure fluctuations. Constant monitoring of his vital signs could help him to reduce his anxiety, decrease the need for routine visits to medical facilities, and also detect early warning features of a possible impending event. He has a smart phone with SAAP installed on it and is willing to wear biosensors to measure his vital signs. The data is wirelessly sent to his mobile where SAAP detects any changes not only in his vital signs but in any contextual information that is related to the application (e.g. the battery level of the mobile phone). SAAP uses this information to reason about situations in real-time and according to inferred situations, it performs intelligent and cost-efficient analysis of data. When fluctuations of vital signs are within a specified "acceptable" threshold, there is no need for frequent measurement and use of resources can be reduced and moderated. However, when these fluctuations are over the threshold, this "situation" warrants a closer monitoring by the system and more frequent measurements. This type of adaptation requires factoring in both available resources and criticality of health situations.

This paper is structured as follows: Section 2 discusses the related work. Section 3 presents the SAAP architecture. Section 4 describes the Fuzzy Situation Inference (FSI) that enables situationawareness. Section 5 discusses the adaptation engine. Section 6 and 7 describes implementation and evaluation respectively. Finally section 8 concludes the paper and discusses the future work.

\section{Related Work}

Mobile healthcare computing is a new and evolving area of research that exploits the recent development in mobile networks and communications for health monitoring applications. EPI-MEDICS [5] is a large scale European project that provides personal monitoring of ECG signals for early detection of cardiac ischemia and arrhythmia and generating different levels of alarms. Another European project called the MobiHealth project [6] uses 2.5 (GPRS) and 3G (UMTS) technologies to integrate all the sensors and actuators into a wireless network called Body Area Network (BAN). The project of ubimon (Ubiquitous Monitoring Environment for Wearable and Implantable Sensors) [7] aims to provide continuous management of patients mainly focusing on sensors and wireless technology rather than data analysis techniques. Personalization is another area of focus in developing mobile health monitoring applications that has been studied in [8].

Context-awareness is one of the key requirements of health monitoring systems that enables autonomous operations without patient's intervention and enhances decision making of healthcare professionals on patient 
SAAP layer is built on the top of the data stream mining algorithms running on mobile devices and provides them with situation-aware adaptation. The next section discusses the FSI technique. 
These techniques integrate fuzzy logic into the CS reasoning methods to provide another aspect of uncertainty (i.e. uncertainty of situations and delta changes of context) in the computation of confidence value for the occurrence of a situation.

The situation reasoning techniques of CS are based on four heuristics that are introduced to manage uncertainty in pervasive computing environments. These heuristics are as follows: i) relative weights of context attributes and confidence level of values; ii) sensors' inaccuracy; iii) symmetric and asymmetric context attributes; iv) and partial and complete containment of symmetric context attributes. Table 1 depicts reasoning methods of CS, their FSI equivalent that are combined with fuzzy logic and their underlying heuristics and theoretical concepts.

The next subsections discuss each heuristic and reasoning technique in more detail.

4.3.1. Weights and contribution level. The first reasoning technique of CS is based on the weights of context attributes and the level of confidence of attributes' values. Weights are assigned to context attributes and represent relative importance of each context attribute for inferring a situation. Level of confidence is assigned to each element and reflects how that element relates to the modeled situation. In this heuristic, the contribution function that computes the contribution level is proposed at a conceptual level and its implementation is later introduced in the second reasoning technique based on sensors' inaccuracy.

In FSI, the concept of weights is associated with linguistic variables (i.e. context attributes). The concept of contribution level is similar to the membership degree of elements in a fuzzy set but they are implemented using membership functions. The result of $\mu(\quad)$ represents a weighted membership degree of and represents the number of conditions in a rule $(1 \leq \mathrm{i} \leq \mathrm{n})$.

\subsubsection{Sensors' inaccuracy.}


values that linguistic variables take are not numeric (i.e. these values are called terms that represent fuzzy sets), the concept of symmetric and asymmetric concepts are applied to the values of fuzzy sets associated with linguistic variables. 
time. Controller that is a subcomponent of AE makes decisions on which strategy needs to be performed according to these thresholds. These nine cases are presented in Table 2. We have allocated the adaptation strategies according to these nine cases. When resources are critical it means that the mobile device 
strategy. When the adaptation cases 5 or 6 occurs, resource-aware and situation-aware adaptation strategies each compute different values according to resource availability and occurring situations respectively. Therefore there is a trade-off between the results of these two strategies. Hybrid adaptation strategy addresses this issue by computing the average value of parameter based on the results of the two strategies and criticality values of the situation and resource as follows:

4)

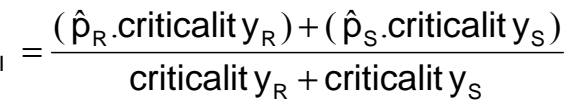

Having discussed the theoretical framework of our work, the following section presents the implementation and evaluations we have performed.

\section{Implementation}

We have implemented a prototype of health monitoring application based on FSI in J2ME and deployed it on a Nokia N95 (shown in Figure 3). The prototype reasons about situations of 'normal', 'prehypotension', 'hypotension', 'pre-hypertension' and 'hypertension'. This application can be used by patients who suffer from blood pressure fluctuations. A trapezoidal membership function is used to compute membership degree of context values. Contextual information used includes systolic and diastolic blood pressure (SBP and DBP) and heart rate (HR).

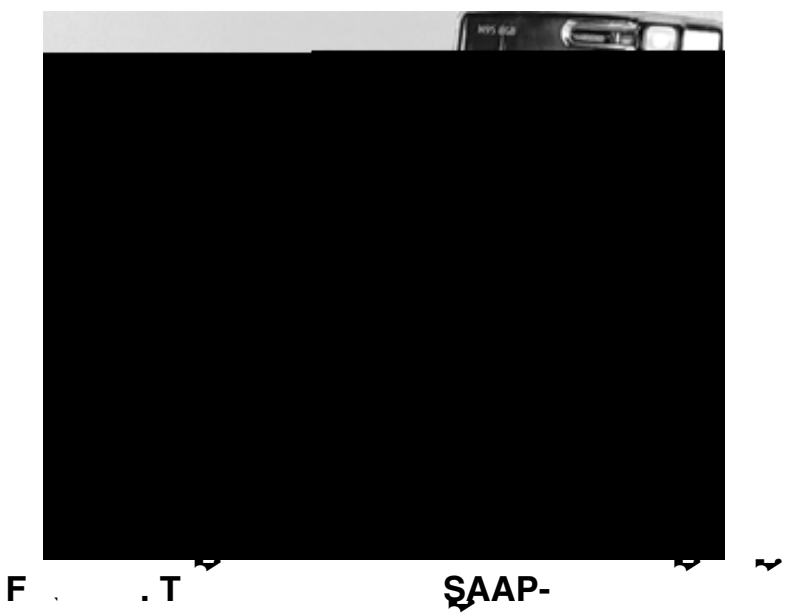

To capture the patient's heart rate, we have used a two lead ECG biosensor from Alive Technologies [23] that transmits ECG signals using Bluetooth to the mobile phone. For the blood pressure, we have used randomly generated data that simulates blood pressure fluctuations. The health monitoring application performs situation reasoning and situation-aware adaptation in real-time on the mobile device using the LWC algorithm. Status bars on the mobile phone displays the level of certainty and confidence in the occurrence of each situation.

The evaluation of FSI and adaptation engine is presented in the next section.

\section{Evaluation}

For evaluation of SAAP, we have performed two evaluations. First evaluation is a comparative evaluation of FSI, CS and Dempster-Shafer and second evaluation focuses on the adaptation of threshold parameter of LWC according to occurring situations.

\subsection{Evaluation of FSI}

To evaluate the FSI model, we have compared the FSI situation reasoning technique to the CS and Dempster-Shafer (hereafter DS) reasoning approaches. The purpose of this evaluation is first to validate the FSI model against a well-known reasoning technique such as DS and a context model developed for pervasive computing environments such as CS. The second objective of the evaluation is to highlight the benefits of the FSI to deal with uncertain situations.

In this evaluation, we have considered situations of 'hypotension', 'normal' and 'hypertension'. These situations are defined using context attributes of systolic blood pressure (SBP) and diastolic blood pressure (DBP) with the scale of 40-170 and 20-150 $\mathrm{mm} \mathrm{Hg}$ and heart rate (HR) with the range of 20-150 bpm.

Table 3 depicts modeling of the three situations in the CS model including the weights of attributes and their corresponding regions of values. Assigned weights are 0.4 for SBP and DBP and 0.2 for HR.

\begin{tabular}{|lll|}
\multicolumn{1}{c}{ T } & \multicolumn{1}{c}{$\mathbf{S}$} & \multicolumn{1}{c}{$\mathbf{S}$} \\
\hline Situation & Context attribute & Region of values \\
Hypotension & $1=$ SBP & $\leq 85$ \\
& $2=$ DBP & $\leq 60$ \\
Normal & $3=$ HR & $\leq 45$ \\
& $1=$ SBP & $>85$ and $\leq 135$ \\
& $2=$ DBP & $>60$ and $\leq 110$ \\
Hypertension & $3=$ HR & $>45$ and $\leq 85$ \\
& $1=$ SBP & $>135$ \\
& $2=$ DBP & $>110$ \\
& $3=$ HR & $>85$ \\
\hline
\end{tabular}

The modeling of the three situations in the FSI model is presented in Table 4. Weights of conditions for the FSI rules conform to the weights used in CS. 
$\mathbf{T}$

Situation represented

- $\mathrm{FSI}-2=\mathrm{DB}$

rules

$3=\mathrm{HR}$

FS

Terms

low, normal, high

low, normal, high

slow, normal, fast 
the values and are not able to reflect the gradual evolution of one situation to another situation. When the value of context attributes decreases or increases, its membership degree also increases and decreases accordingly and gradually. This enables FSI to provide 
with The Eighth Pacific-Asia Conference on Knowledge Discovery and Data Mining, Sydney, Australia, 2004.

[2] M.M. Gaber, S. Krishnaswamy, and A. Zaslavsky, "Resource-Aware Mining of Data Streams", Journal of 